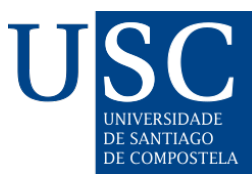

\title{
Barcelona como laboratorio de innovación democrática (2015-2020)
}

\section{Barcelona as a laboratory of democratic innovation (2015-2020)}

\author{
Jordi Bonet Martí ${ }^{1, a,}{ }^{*}$, Mercedes Serrano Miguel $^{2, \mathrm{~b},{ }^{* *}}$ \\ ${ }^{1}$ Universitat de Barcelona, España \\ ${ }^{2}$ Universitat de Barcelona, España \\ a jordi.bonet@ub.edub mercedes.serrano@ub.edu
}

Recibido: 15/10/2021; Aceptado: 28/12/2021

\section{Resumen}

Este artículo tiene por finalidad conocer el diseño e implementación de las nuevas formas de coproducción de gobernanza relacional y democrática que se han desarrollado en la ciudad de Barcelona durante el periodo 2015-2020 coincidiendo con el primer mandato del gobierno municipal de Barcelona en Comú. Para ello, nos centramos en el análisis de tres procesos de innovación democrática -la gestión cívica de equipamientos públicos, la producción de comunes urbanos y la democratización de la innovación digital- que ejemplificamos a través de tres casos de estudio: el Centro LGTBI de Barcelona, el proyecto cooperativo Can Batlló y el Canódromo - Ateneo de Innovación Digital y Democrática. Tal y como se evidencia en la comparación desarrollada, estos tres modelos suponen un cambio respecto a los modelos tradicionales de gestión pública al permitir articular una nueva relación entre movimiento e institución, su orientación hacia la autogestión y su articulación con un nuevo marco normativo, dentro del marco conceptual de la cooperación público-comunitaria.

Palabras clave: Innovación social; comunes urbanos; gestión cívica; democratización; innovación digital.

\begin{abstract}
The purpose of this article is to know the design and implementation of the new forms of co-production of relational and democratic governance that have been developed in the city of Barcelona during the period 2015-2020, coinciding with the first mandate of the local government of Barcelona en Comú. To do this, we focus on the analysis of three democratic innovation processes - the civic management of public facilities, the production of urban commons and the democratization of digital innovation - which we exemplify through three case studies: the Barcelona LGTBI Center, the cooperative project Can Batlló and the Canòdrom Ateneo de Innovación Digital y Democrática. As evidenced in the comparison developed, these three models represent a change with respect to the traditional models of public management by allowing the articulation of a new relationship between movement and institution, its orientation towards self-management and its articulation with a new normative framework, within of the conceptual framework of public-community cooperation.
\end{abstract}


Keywords: Social innovation; urban commons; civic management; democratization; digital innovation.

\section{Sumario}

1. Modelos de gestión alternativos

2. Barcelona y la innovación social

3. El Centro LGTBI y la gestión cívica

4. Los comunes urbanos y Can Batlló

5. La innovación democrática y el Canòdrom

6. Comparación de los tres modelos de innovación

7. Discusión y conclusions

\section{Summary}
1. Alternative management models
2. Barcelona and social innovation
3. The LGTBI Center and civic management
4. The urban commons and Can Batlló
5. Democratic innovation and the Canòdrom
6. Comparison of the three innovation models
7. Discussion and conclusions

\section{MODELOS DE GESTIÓN ALTERNATIVOS}

La prestación de servicios públicos se ha basado bien en los modelos de gestión directa, en que la administración pública se ocupa de la prestación integral del servicio, bien en los de gestión indirecta, en que la prestación se externaliza al sector privado (del Guayo, 2004). Aunque estos modelos son los más comunes al abordar los servicios públicos locales en los últimos años han emergido otros modelos de gestión que tienen como elemento distintivo la incorporación de la ciudadanía y del movimiento asociativo en los procesos de co-diseño y co-producción de servicios públicos (Pastor Albaladejo, 2014). A diferencia de los modelos anteriores, que han sido profusamente abordados por la literatura sobre gestión pública, los modelos emergentes se caracterizan por su condición abierta, contextual y procesual, de modo que el estudio de su implementación y desarrollo en entornos locales es relevante para el campo de los estudios urbanos y la gestión pública (Pont Vidal, 2016).

Tradicionalmente, la gestión directa ha sido identificada como el modelo propio de la administración pública, siguiendo el tipo ideal de la racionalidad burocrática (Weber, 1922; 2014). Sin embargo, a partir de la década de los 80 del siglo pasado, empiezan a difundirse otros modelos basados en la gestión indirecta, mediante la creación ad hoc de diferentes unidades especializadas (organismos autónomos, sociedades mixtas, sociedades mercantiles) o la adjudicación de servicios, en el contexto de difusión del paradigma de la nueva gestión pública (Hood, 1991).

La incorporación de estos nuevos procedimientos ha tenido por objetivo lograr una mayor competitividad, eficiencia y efectividad en la satisfacción de las demandas sociales y su desarrollo ha coincidido con la crisis del estado de bienestar keynesiano y la necesidad de crear nuevos modelos de gestión a fin de desburocratizar las administraciones públicas (Brugué y Gomà, 1994). En la arena de los gobiernos locales, su adopción ha contribuido a la expansión del modelo de 
gerencialismo público (Pascual, 2016) que se caracteriza por establecer una división neta entre el plano político y administrativo mediante la creación de estructuras gerenciales y la incorporación de instrumentos (auditorías externas, cuadros de mando integrales, catálogos de servicios...) derivados del mundo empresarial.

Por otra parte, la crisis del modelo tradicional de provisión de servicios centrado en el estado ha conllevado una creciente complejización de las relaciones entre el estado, mercado y sociedad, que ha sido teorizada desde las ciencias sociales bajo el paradigma de la gobernanza (Mayntz, 1993; Scharpf, 1994). En este sentido, si la nueva gestión pública se orienta principalmente a los procesos orientados a la modernización de las formas y procesos de gestión, la gobernanza sitúa el foco en el paso del gobierno tradicional, centrado en la acción y capacidad del estado, al gobierno en red, que se ejerce a través de las relaciones y compromisos entre diferentes actores. Si bien, gobernanza y nueva gestión pública no son conceptos sinónimos, ni en el plano conceptual ni en el plano operativo, ha existido una tendencia a hacerlos converger (Klijn, 2012), tal y como se evidencia en las recomendaciones de organismos transnacionales como el Banco Mundial o el Fondo Monetario Internacional para la modernización de las administraciones públicas.

Sin embargo, tanto la nueva gestión pública como la gobernanza han sido objeto de críticas. Dunleavy y Hood (1995) consideran que la adopción indiscriminada por parte de las administraciones de la nueva gestión pública puede afectar negativamente al desempeño de las capacidades fundamentales del estado y de la política. Más recientemente, Levesque (2013) subraya los límites derivados de generar un proceso de doble fragmentación en la acción de gobierno: una vertical, que separa lo político y la administración, y otra horizontal, que atomiza las relaciones entre las distintas unidades especializadas. En relación con la gobernanza, Swyngedouw (2005) señala los riegos que esta implica para la representatividad, la transparencia y la rendición de cuentas. De acuerdo con estas críticas, la difusión de la nueva gestión pública y el arraigo de una concepción neoliberal de la gobernanza entraña el riesgo de una despolitización y desdemocratización de lo público, al quedar este sometido a los criterios del mercado, ampliando de este modo la distancia que separa las instituciones de la ciudadanía (Bonet-Marti, 2021).

En este sentido, se han desarrollado modelos alternativos que inciden en la necesidad de completar la democracia representativa con una democracia participativa que permita una mayor capacidad de deliberación y decisión de la ciudadanía en los asuntos públicos (Barber, 1984). A su vez, frente a los criterios meramente instrumentales que guían la nueva gestión pública, estos modelos alternativos acostumbran a optar por otorgar un mayor reconocimiento al criterio de valor público (Moore, 1998), concebido como un proceso de generación de valor diferenciado del privado y que incorpora tanto los beneficios sociales generados, así como el valor de los procesos democráticos y participativos a través de los cuales este valor ha sido producido.

Estos modelos cobran relevancia a raíz de la crisis social, económica y financiera de 2008, cuando se evidencia que los modelos clásicos de gobernación son incapaces de generar respuestas satisfactorias a los desafíos sociales más complejos, multidimensionales, multifactoriales y multinivel (Hernández-Ascanio; Tirado-Valencia y Ariza-Montes, 2016) asociados al desarrollo del capitalismo informacional (Castells, 1997), y más recientemente, a la crisis climática. A su vez, también cobran relevancia las demandas de la ciudadanía para obtener una mayor capacidad de control y participación en las decisiones públicas (Bekkers, Tummers y Voorberg,2013) como respuesta a las crisis de los modelos liberales de democracia vaciados de su componente popular (Mair, 2013) y sometidos a la elitización de las decisiones políticas condicionadas a los intereses económicos (Crouch, 2004). 
De hecho, la crisis de 2008 ha supuesto la aparición de dos ejes de confrontación en el ámbito social que aún perduran hoy en día. Por una parte, la relación entre democratización y desdemocratización (Tilly, 2007) que cobra relevancia en relación con la implementación de las políticas de austeridad y que luego se acentúa con el auge de los populismos de derecha. Por otra parte, la crisis evidencia también la emergencia de la tensión entre la mercantilización y las formas de protección con las que la sociedad responde a esta tendencia, de acuerdo con la teoría del doble movimiento descrito por Polanyi (1944; 2016).

En este artículo abordamos el desarrollo de estos modelos alternativos desde el prisma de su relación con los movimientos sociales en su rol de co-productores de servicios públicos en el contexto de la ciudad de Barcelona durante el periodo 2015-2020. Tradicionalmente, la relación entre movimientos sociales e institución se ha desarrollado a partir de la dualidad oposición/ radicalización - institucionalización; sin embargo, siguiendo la línea de trabajos desarrollados por Ibarra, Martí y Gomà (2008) y su continuación en Ibarra, Gomà, Martí y González (2018), en este artículo nos interesa centrarnos en la capacidad que disponen movimientos ciudadanos y sociales para coproducir políticas, gestionar servicios públicos y participar críticamente en la gobernanza urbana.

Cabe señalar que esta implicación de los movimientos sociales en la gobernanza urbana no es nueva, y que disponemos de ejemplos anteriores que van desde la implementación de los presupuestos participativos de Porto Alegre (Sousa Santos, 2003) hasta el uso estratégico por parte de los movimientos sociales alemanas de las Bürgerinitiativen (iniciativas ciudadanas) a fin de impulsar cambios en las políticas públicas (della Porta, 1995). No obstante, consideramos que es en el marco del contexto socioeconómico derivado la crisis cuando asistimos a una multiplicación y difusión de nuevas prácticas ciudadanas (Pradel y García, 2018) que bajo la forma de redes comunitarias y prácticas autoorganizadas desarrollan una contestación a la agenda neoliberal y de austeridad (Davies y Blanco, 2017). Estas iniciativas críticas constituyen una nueva institucionalidad de lo común (Laval y Dardot, 2015) que promueve un nuevo marco de gobernanza democrática (Pascual, 2016), de carácter híbrido y relacional (Grau, Iñíguez-Rueda y Subirats, 2011) y que emerge como una nueva forma de gobernación de los asuntos públicos adaptada a sociedades complejas (Subirats, 2011).

Dentro de este proceso de cambio, cabe señalar la centralidad que adquiere el paradigma de los comunes construidos en el ámbito de la proximidad (Subirats, 2016), como instrumento de generación de una agenda de políticas urbanas orientada al derecho de la ciudad (Blanco, Gomà y Subirats, 2018). Aún y no tratarse de un concepto nuevo, el estudio de los bienes comunes se había ya abordado desde la economía y el derecho, si bien en la mayoría de casos eran concebidos como un residuo jurídico de las sociedades preindustriales o como un tipo de bienes cuya gestión comunitaria podía ser disfuncional para su supervivencia, tal y como recoge el argumento de la tragedia de los comunes de Hardin (1968); no obstante, esta concepción comenzó a cambiar en el campo académico a partir de la publicación de los hallazgos de Ostrom (1990). De acuerdo con los estudios de la economista estadounidense, las instituciones comunitarias encargadas de recursos de uso común podrían alcanzar una gestión más robusta y eficiente que la desarrollada por el estado o el mercado. Esta línea de argumentación se ha visto refrendada por el desarrollo de los comunes digitales, también llamados comunes emergentes, y que se vinculan al modelo de producción procomún entre iguales (Benkler, 2015) y a las comunidades de creación online (Fuster, 2010), como las comunidades FLOSS (free/libre and open-source software) o la creación digital abierta. 
Los comunes digitales se refieren a la producción y compartición de recursos comunes a partir de "la colaboración (en ocasiones, a gran escala) de individuos a través de plataformas online, en las que no hay una planificación centralizada del proceso. En estas plataformas, la participación es eminentemente abierta, autónoma y voluntaria" (Fuster; Subirats; Berlinguer; Martínez; Salcedo, 2015, p. 14). Una de las características de los comunes digitales es que no están afectados por la ley de rendimientos decrecientes de manera que el coste marginal de producir una unidad adicional de un producto es tendencialmente igual a cero (Rifkin, 2014), lo que conlleva que estos puedan ser ampliamente reproducidos, adaptados y mejorados por el conjunto de la sociedad, sin que ello implique un descenso de su rendimiento.

Siguiendo la senda abierta por Ostrom y los comunes digitales, en los últimos años han cobrado relevancia los comunes urbanos. Méndez, Hamou y Aparicio (2021, p. 7) los definen como "procesos políticos de apropiación y transformación por parte de la comunidad organizada de los recursos y servicios públicos necesarios para la reproducción social”. En este sentido, bajo la denominación de comunes urbanos podemos incluir todo un conjunto de prácticas orientadas al sostenimiento de la vida: desde la defensa de los servicios públicos hasta la reapropiación del espacio público frente a su colonización por el mercado (Observatori Metropolità de Barcelona, 2014). Esta condición de prácticas sociales es subrayada por Harvey (2017, p. 116) cuando afirma que "los bienes comunes no deben considerarse pues como un tipo particular de cosas o activos y ni siquiera de bienes sociales, sino como una relación social, inestable y maleable entre cierto grupo social autodefinido y los aspectos de su entorno social o físico, existente o por ser creado, considerada sustancial para la vida y su pervivencia. De hecho, existe una práctica social de comunalización que produce o establece una relación social con un determinado bien común".

De esta manera, la perspectiva de los comunes urbanos trasciende el concepto de bienes comunes teorizado por Ostrom, al incorporar la idea de comunalización (commoning) entendida como proceso dinámico de producción de lo común que no está sujeto ni a la lógica del mercado ni a la del estado, por lo que la noción de comunes urbanos apunta hacia una repolitización de lo procomún, entendida como la creación de un espacio en disputa frente a las lógicas de apropiación de lo privado y lo estatal que ya fueron denunciadas por el manifiesto Los Nuevos Cercamientos elaborado por el Midnight Notes Collective (Federici, 2020).

\section{BARCELONA Y LA INNOVACIÓN SOCIAL}

En las últimas décadas, la región metrópolitana de Barcelona se ha constituido en un territorio referente para la innovación social, tanto por su histórico acumulado de prácticas de creatividad social, como por la acumulación de procesos innovadores en el campo de la participación ciudadana en el ámbito local (Bonet-Marti, 2012).

Si bien el concepto de innovación social es de reciente aparición, las prácticas que describe tienen un mayor recorrido; de hecho, en el caso de la metrópolis barcelonesa pueden asociarse a la corriente mutualista, asociativa y pedagógica que configuró una parte del movimiento obrero catalán desde la segunda mitad del siglo XIX hasta la Segunda República. De hecho, ha sido en la región barcelonesa, y con especial incidencia, los barrios y áreas urbanas más desfavorecidas (Blanco; Cruz; Martínez y Parés, 2016) donde han cristalizado una miríada de iniciativas ciudadanas como: la creación de la Plataforma de Afectados por la Hipoteca (PAH), de redes de crianza 
compartida, los sindicatos de inquilinos, de huertos urbanos o de espacios de co-creación artística y tecnológica (Blanco y Gomà, 2016).

Por otra parte, desde la recuperación de la democracia municipal, el Ajuntament de Barcelona, así como otros gobiernos locales de la región metropolitana, ha destacado por su capacidad de impulsar políticas de descentralización y de participación ciudadana (Gomà y Rebollo, 2001). Estas políticas que tienen su origen en los primeros gobiernos municipales surgidos tras la recuperación de la democracia, se desarrollan en mayor medida tras la aprobación de las normas de participación ciudadana de 2002 y del reglamento de participación ciudadana de 2008 que establece un sistema de participación organizado sectorialmente y orientado principalmente a las entidades (consejos municipales) como territorialmente (consejo de ciudad, y consejos de barrio y distrito) dónde tienen cabida los ciudadanos. Esta tendencia democratizadora en el ámbito local se acentúa con la aprobación del nuevo reglamento de participación ciudadana de 2017, a través del desarrollo de instrumentos como la iniciativa y la consulta ciudadana que permitirían una mayor implicación de la ciudadanía y el tejido asociativo en el gobierno de la ciudad. No obstante, esta dinámica de profundización ha sido obstaculizada judicialmente a raíz de la sentencia 874/2019 de la sala del contencioso del Tribunal Superior de Justicia de Cataluña que ha anulado el reglamento en base a no respetar "el régimen local básico establecido" a raíz del recurso contra la celebración de una multiconsulta en que la ciudadanía de Barcelona tenía que pronunciarse acerca de la propuesta de municipalización del servicio de abastecimiento de agua.

Sin embargo, si hay un hito clave que marca la diferencia en las formas de entender la participación ciudadana y las relaciones de la institución con los movimientos sociales de la ciudad, esté ha sido el acceso al gobierno de la ciudad de la plataforma de confluencia ciudadana Barcelona en Comú liderada por la activista de la PAH Ada Colau tras las elecciones municipales de 2015. Si bien, tras las elecciones municipales de 2015, diversas plataformas de confluencia ciudadana constituidas post 15M (Ahora Madrid, Barcelona en Comú, Marea Atlántica, Compostela Aberta, Zaragoza en Común, Por Cádiz si se puede...) consiguen acceder al gobierno de distintas ciudades españolas, tan solo las de Barcelona y Cádiz conseguirán los apoyos políticos y electorales suficientes para revalidar su gobierno en las elecciones municipales de 2019. En este sentido, el caso de estudio de Barcelona nos aparece como social y académicamente relevante no solo por el historial de participación e innovación presente en la ciudad, sino también por el impulso que reciben estas políticas durante el primer mandato del gobierno municipal de Barcelona en Comú y su continuidad en el presente, a diferencia de ciudades como Madrid, en donde la victoria de la derecha en las elecciones ha frenado las políticas de participación.

En este sentido, podemos considerar que el acceso a los gobiernos municipales por parte de las confluencias ciudadanas ha supuesto un cambio en la estructura de oportunidades políticas que ha modificado la relación entre movimientos e institución, y ha acentuado las dinámicas de colaboración por encima de las de conflicto, que habían dominado el periodo anterior, a la vez que ha evidenciado las constricciones normativas e institucionales que limitan desarrollo de procesos de cambio e innovación social (Ibarra; Gomà; Martí y González, 2018).

Si bien, el estudio de los cambios desarrollados en el conjunto del modelo de participación excede los propósitos de este artículo, nos centraremos en los cambios vinculados a la relación entre asociaciones/movimientos e institución en el marco de coproducción de una gobernanza relacional y democrática. En este sentido, nuestro objetivo ha sido el de identificar y analizar el proceso de creación de diferentes modelos de innovación política, social y democrática en el contexto de cambio propiciado por el acceso de Barcelona en Comú al gobierno de la ciudad, centrándonos en 
las políticas de impulso a la innovación y la participación desarrolladas durante su primer mandato (2015-2019). Para ello, nos centramos en un análisis comparado de tres modelos de innovación (la gestión cívica, los comunes urbanos y los ateneos de innovación democrática) que vinculan la acción política municipal con prácticas, demandas y marcos conceptuales impulsados desde los movimientos.

\section{EL CENTRO LGTBI Y LA GESTIÓN CÍVICA}

Aunque el inicio de la gestión cívica es anterior a la llegada de Barcelona en Comú al gobierno municipal, es durante el periodo 2015-2019 cuando este modelo conoce su mayor desarrollo e implementación, a partir de la aprobación del Modelo de Bases (Ajuntament de Barcelona, 2015) y posteriormente del Programa de Patrimonio ciudadano (Ajuntament de Barcelona, 2017), convirtiéndose de esta manera en el modelo predominante en la gestión de nuevos equipamientos cívicos y barriales, sustituyendo progresivamente al modelo de gestión indirecta basado en la adjudicación mediante un contrato de servicios. Actualmente, existen más de 70 equipamientos en la ciudad gestionados bajo el modelo de gestión cívica o ciudadana (Castro y Forné, 2021) frente a los 14 equipamientos y servicios que funcionaban bajo este modelo de gestión en 2012 (Plataforma Gestió Ciutadana, 2012).

La gestión cívica se encuentra recogida en el artículo 34 de la Carta Municipal de Barcelona que reconoce a "las entidades, organizaciones y asociaciones sin ánimo de lucro la posibilidad de ejercer competencias municipales o participar en nombre del ayuntamiento en la gestión de servicios o equipamientos la titularidad de los cuales corresponde a otras administraciones públicas" (Ajuntament de Barcelona, 1998, p. 20). La existencia de este marco normativo con rango de ley (Ley 22/1998, de 30 de septiembre) es el que permite al Ajuntament de Barcelona desarrollar este modelo sin necesidad de remitirse a la Ley 9/2017, de 8 de noviembre, de Contratos del Sector Público.

No obstante, aunque la gestión cívica esté amparada por la Carta Municipal, no ha sido hasta la aprobación del modelo de bases que se genera un marco regulativo para su instrumentación y unos criterios claramente definidos; mientras su implementación anterior estaba solo limitada a un conjunto de equipamientos (ateneos y casales de barrio, centros cívicos y polideportivos) en territorios con una elevada densidad asociativa.

En este sentido, la demanda de dotar de mayor seguridad jurídica a la gestión cívica ha sido una reivindicación exigida por el movimiento asociativo barcelonés, tanto por el Consejo de Asociaciones de Barcelona (CAB) y más específicamente por la Plataforma d'entitats per a la gestió cívica constituida en 2009 y que tenía por objetivo modificar el marco legal que regía la adjudicación de equipamientos públicos a fin de facilitar la participación de las entidades (Sánchez Belando, 2015). Esta plataforma, posteriormente denominada Plataforma de Gestió Ciutadana (2012), actualmente aglutina entidades de la ciudad que gestionan servicios y equipamientos, o aspiran a gestionarlos, de acuerdo con el modelo de gestión cívica.

Esta demanda ha sido en parte satisfecha con la aprobación del Modelo de bases de la gestión cívica de equipamientos, para actividades y servicios municipales que ha permitido su expansión a un mayor número de equipamientos de la ciudad. Si bien el Modelo de bases se aprueba dos meses antes de las elecciones municipales de 2015, ha sido durante el gobierno de Barcelona en Comú cuando se ha desplegado, dándole mayor impulso y fuerza jurídica a través de su incorporación 
en el Programa de Patrimonio Ciudadano de uso y gestión comunitaria. Este marco regulatorio ha permitido un cambio en la cultura organizativa del ayuntamiento, evitando la arbitrariedad en la adjudicación de servicios y aumentando la equidad en la concurrencia entre sector privado y sector comunitario.

El modelo de gestión cívica desarrollado por el Ajuntament de Barcelona se basa en la incorporación de entidades sociales y redes ciudadanas a la gestión de servicios públicos a través de mecanismos de cesión, cogestión o creación de nuevos servicios participados por la ciudadanía. En muchos casos, el modelo de gestión ha supuesto el reconocimiento como servicio público de prácticas ciudadanas ya existentes en el territorio formalizadas a través de la firma de un convenio con la administración, mientras en otros, supone la creación de un equipamiento/servicio ex novo, tal y como ha sucedido con el Centro LGTBI de Barcelona.

El centro LGTBI de Barcelona se crea en enero de 2019 como un espacio de referencia de la diversidad sexual y de género en la ciudad y adopta el modelo de gestión cívica. La iniciativa de creación del centro surge del Consejo Municipal LGTBI, consejo consultivo y órgano de participación sectorial que se constituye en 2004 con el objetivo de fomentar la igualdad de derechos, libertades y reconocimiento social de las personas LGTBI. Un elemento diferencial del equipamiento en relación con otros que adoptan este modelo de gestión es su condición de equipamiento de ciudad que ofrece servicios integrales a la ciudadanía.

La llegada de Barcelona en Comú al gobierno de la ciudad ha supuesto la priorización de la diversidad sexual y de género en la agenda política urbana a través de la nueva concejalía de Feminismos y LGTBI a partir de la aprobación del Plan Municipal para la Diversidad Sexual y de Género. Medidas para la igualdad LGTBI (2016-2020), en que se incluía la creación del centro LGTBI. Para ello, en 2016 se desarrolla un proceso participativo para definir el nuevo Centro Municipal de Recursos LGTBI de la ciudad en dos fases: en la primera, se recogen las propuestas de las entidades LGTBI de la ciudad, con especial atención a los servicios y programas que las entidades consideraban necesarios. En la segunda fase, se crea un grupo de trabajo mixto entre entidades y equipo técnico de la concejalía a fin de concretar la estructura y normas de funcionamiento del centro.

El modelo de gestión del centro se basa en un convenio con la Plataforma de Entidades LGTBI de Catalunya que está integrada por 13 entidades referentes en el sector. A partir de allí, se constituye un Consejo del Centro que funciona como órgano de gestión y en él se encuentran representados la concejalía de Feminismos, el consejo municipal LGTBI, la dirección del centro y las entidades que participan del mismo. En este sentido, el modelo de convenio supone un cambio respecto a la gestión de otros equipamientos municipales sectoriales que operaban a través de un contrato de adjudicación a una entidad del tercer sector. Actualmente, el Centro se encuentra en proceso participativo de elaboración de su reglamento de funcionamiento interno que servirá para acabar de definir la gobernanza del centro.

Desde su inauguración, el centro desarrolla servicios de información y dinamización, la acogida personal y familiar, el asesoramiento psicológico, la orientación legal y jurídica, el asesoramiento laboral, la información socio-sanitaria y un servicio específico de atención a personas trans e intersexuales. A su vez opera como espacio de acogida de entidades que prestan servicios específicos a la comunidad LGTBI, entre las que se incluye el Observatorio contra la Homofobia, además de operar como espacio de reuniones, encuentros, charlas y exposiciones. 


\section{LOS COMUNES URBANOS Y CAN BATLLÓ}

Si en el modelo de gestión cívica, el protagonismo se centra en la capacidad de co-gestión asociativa de equipamientos, el modelo de comunes urbanos se asocia a la generación de instituciones de lo común con una voluntad explícita de plantear una alternativa socio-económica al modelo capitalista neoliberal. La incorporación de los comunes urbanos a las políticas públicas se produce con la llegada al gobierno de la ciudad de Barcelona en Comú.

Una de las iniciativas más ambiciosas en esta dirección ha sido la aprobación del Programa de Patrimonio Ciudadano (Ajuntament de Barcelona, 2017) cuya finalidad es fomentar la colaboración público-comunitaria en la ciudad de Barcelona, mediante la promoción de nuevas formas de interacción entre la institución pública municipal y las iniciativas ciudadanas de base, a partir del reconocimiento del derecho a la gestión y al uso comunitario del patrimonio público por parte de la ciudadanía. Es decir, frente al modelo de cooperación público-privada se apuesta por la creación de una nueva institucionalidad democrática que tiene por objetivo "garantizar y facilitar la existencia de espacios auto-gestionados y con autonomía que puedan generar sus propias formas de valorizar el trabajo y la función pública" (Castro y Forné, 2021, p. 14).

Entre las experiencias incluidas en el Programa destacan la elaboración de un censo de locales y parcelas municipales y la creación del Balance Comunitario, a fin de disponer de nuevos indicadores que permita valorar y evaluar de forma óptima, teniendo en cuenta el valor añadido de los procesos comunitarios y dejando fuera indicadores de carácter económico y mercantil. El Balance Comunitario tiene por finalidad convertirse en una herramienta de rendición de cuentas que permita conocer el valor que un determinado servicio aporta a la ciudad y a su vez, en una herramienta de seguimiento y evaluación que facilite que la cesión de patrimonio público continúe en manos de proyectos dinámicos, en vez de en entidades que languidecen.

El Programa de Patrimonio Ciudadano se basa en tres líneas de actuación:

1. La Gestión comunitaria de bienes municipales a partir de la cesión de patrimonio municipal.

2. La Gestión comunitaria de equipamientos, a partir de la cesión de equipamientos municipales y de dotación económica para el desarrollo de las actividades.

3. El apoyo y acompañamiento a servicios de iniciativa ciudadana, que supone abrir una línea de financiación para proyectos de gestión comunitaria que hagan provisión de servicios no obligatorios de interés público.

También ha supuesto la elaboración de un censo de solares y locales municipales con el objetivo de crear un catálogo para la cesión de patrimonio municipal, y la creación de un órgano de gobernanza con participación ciudadana entre los proyectos comunitarios, equipamientos y Ajuntament.

En este marco cabe ubicar el desarrollo del proyecto de Can Batló. Se trata de un antiguo complejo industrial ubicado en el barrio de Sants de Barcelona con una superficie de nueve hectáreas que originalmente estaba destinado a convertirse en zona verde y equipamientos. Tras 43 años de lucha vecinal, y la campaña "Tic Tac Can Batlló" que culmina con la ocupación vecinal del recinto en 2011, lo que permite la creación de los primeros equipamientos cívicos en el recinto (Sánchez Belando, 2021), en 2019 el Ayuntamiento cede cerca de 1,3 hectáreas sean gestionadas por la entidad Espacio Comunitario y Vecinal Autogestionado de Can Batlló, una 
entidad instrumental creada con este fin, por un periodo de 30 años prorrogable hasta un máximo de 50 años a fin de desarrollar actividades cooperativas y vecinales, muchas de las cuáles ya venían realizándose anteriormente en el recinto, aunque sin la seguridad jurídica otorgada por el convenio de cesión. Así mismo, se crea una comisión de seguimiento paritaria con el Ayuntamiento y la entidad instrumental se compromete a entregar al ayuntamiento un balance de su retorno social al territorio, a la vez que se ha constituido una comisión de seguimiento a fin de decidir las inversiones necesarias para acabar de acomodar el recinto.

El Balance Comunitario es una herramienta de autodiagnóstico y de rendición de cuentas generada por la Red de Economía Social (XES) y la Red de Espacios Comunitarios (XEC) y el Programa de Patrimonio Ciudadano del Ayuntamiento de Barcelona para permitir la rendición de cuentas y la mejora de los procesos de gestión y autogestión comunitaria tanto a nivel de entidad como de proyecto (XES, 2021). En este sentido, el convenio implica que la entidad instrumental se encargará de realizar un balance comunitario del proyecto cada dos años.

Actualmente, Can Batlló alberga diferentes proyectos como Coopolis - Ateneo Cooperativo de Barcelona, la escuela cooperativa y autogestionada Arcadia (Col-lectiu de mestres tècnics de l'Arcàdia, 2018) y que tiene por objetivo acoger las etapas del segundo ciclo de educación infantil, primaria y ESO; el espacio cultural Bloc Onze que acoge diferentes espacios culturales del recinto como la Biblioteca Popular Josep Pons, una cantina cooperativa, un espacio polivalente de encuentro, un auditorio, taller de artes plásticas, escénicas y audiovisuales, salas para exposiciones temporales y representaciones temporales. Además, cabe señalar la creación de la cooperativa de vivienda en cesión de uso La Borda (Cabré y André, 2018).

\section{LA INNOVACIÓN DEMOCRÁTICA Y EL CANÒDROM}

La digitalización está cambiando nuestras formas de producir, consumir, gobernarnos y relacionarnos. Actualmente, la innovación en el ámbito digital está presente en numerosos ámbitos: empresa, gobierno, administración pública, educación y ciudades. Sin embargo, la innovación digital se encuentra también en el origen de nuevas formas de desigualdad y de discriminación: expansión de la brecha digital por edad, género y recursos socioeconómicos; restricción de acceso a las patentes de software; efectos discriminatorios derivados del uso de algoritmos, intromisión en la privacidad y proliferación de discursos de odio y falsas noticias en las redes sociales. Es en este sentido, diferentes autores han apuntado la necesidad de democratizar la innovación digital desde una perspectiva alejada del determinismo tecnológico y el economicismo neoliberal, que apueste por una democratización del acceso y el desarrollo de la tecnología, su contribución al bienestar social y al empoderamiento ciudadano y la promoción de los comunes digitales.

El gobierno municipal de Barcelona desde 2015 ha puesto el foco en la necesidad de democratizar la innovación digital. En este sentido, cabe señalar las líneas de trabajo emprendidas por el ayuntamiento de Barcelona en esta primera etapa como el Plan Barcelona Digital (Ajuntament de Barcelona, 2016a), la medida de gobierno para una gestión ética y responsable de los datos (Ajuntament de Barcelona, 2018) y el Plan Estratégico para la Dirección de Investigación, Desarrollo e Innovación de la Participación (Ajuntament de Barcelona, 2016b) que depende de la concejalía de Participación y Distritos.

En este contexto, cabe señalar la creación del Canódromo -Ateneo de Innovación Democrática, un espacio ubicado en el barrio de Congrés-Indians inaugurado en diciembre de 2020 y que 
tiene por función aproximar la cultura digital y la innovación democrática al territorio y convertirse en espacio de encuentro para comunidades organizadas, proyectos sociales, centros de investigación y ciudadanía. El espacio ocupa las instalaciones del antiguo Canódromo de la Meridiana que cerró sus puertas en 2006, convirtiéndose en un espacio municipal el año 2010. Desde entonces, se plantearon diferentes proyectos vinculados a la innovación digital y las nuevas tecnologías, presentándose en 2016 como un parque de investigación creativa que operase como una incubadora de empresas orientadas a la producción de videojuegos, lo que ocasionó quejas por parte del movimiento vecinal por su carácter cerrado al territorio en una zona falta de equipamientos.

Paralelamente, desde la Dirección de Innovación se plantea la necesidad de dotar de un espacio físico a la comunidad que ha ido fraguándose alrededor del proceso de creación y desarrollo de la plataforma de participación ciudadana decidim.barcelona. En 2020, coincidiendo con el fin de la concesión previa, la Dirección de Innovación democrática decide convertir el Canódromo en un nuevo espacio de experimentación orientado a fomentar la innovación y la democratización digital. Para ello, se convocó un concurso de adjudicación que acaba ganando Colectic, SCCL, cooperativa tecnológica que ofrece servicios para la transformación social y la inclusión digital y que se encarga de la gestión del equipamiento. Este cambio del modelo gestión supone el abandono del modelo de incubadora empresarial y su transformación en un espacio de consolidación de las políticas públicas de innovación democrática a través de la tecnología, abierto a la participación del barrio del Congrés i Els Indians.

A pesar de tratarse de un espacio de reciente creación, en un contexto afectado por las restricciones derivadas de la pandemia de la COVID-19, es pertinente apuntar su innovador modelo de gobernanza. Si bien, su gestión no responde al modelo de gestión cívica al basarse en un contrato de adjudicación y no a un convenio, la gobernanza del del equipamiento se ha diseñado participativamente. Actualmente, la gestión de las actividades se canaliza a través de la Taula Canòdrom, que cuenta con la participación del ayuntamiento, Colectic los proyectos residentes, si bien tiene la voluntad de abrirse en un futuro a las entidades del territorio y a los proyectos afines que utilizan el espacio, pero no son residentes. También existe la plataforma decidim.canodrom en la que puede participar cualquier persona mayor de 16 años que se registre en la plataforma online y que puede participar en la gobernanza a través de las comunidades virtuales de la plataforma y los plenarios abiertos; y finalmente, la Taula Comunitaria formada por trece entidades del barrio Congrés Indians y que tiene como objetivo la construcción de sinergias entre el equipamiento en el territorio. De esta manera, se asegura que las actividades de innovación desarrolladas en el equipamiento se encuentren enraizadas con el tejido comunitario. Por ejemplo, durante la Bienal de Ciencia organizada por el Ayuntamiento de Barcelona se desarrollaron actividades orientadas a utilizar el videojuego Minecraft como herramienta para repensar participativamente el diseño del espacio urbano.

La plataforma decidim.canodrom se basa en la plataforma digital ciudadana Decidim elaborada por el Ayuntamiento de Barcelona para potenciar la democracia participativa y que ha al estar creada en código abierto ha permitido su reutilización por distintas administraciones y entidades sociales (Peña-López, 2019).

El espacio opera como un ecosistema de proyectos e iniciativas alrededor de la tecnología crítica, la democracia participativa y la cultura digital, apostando por facilitar la formación y la capacitación de la ciudadanía en torno a la ciencia y la tecnología desde una perspectiva crítica y democrática. Entre las actividades realizadas cabe señalar el espacio de residencias para 
proyectos que trabajen en áreas de tecnología ciencia y democracia desde tecnologías libres y con una perspectiva de soberanía tecnológica, la celebración de talleres, exposiciones, proyecciones, jornadas y eventos ciudadanos como el DecidimFest, jornadas anuales de reflexión sobre las relaciones entre democracia, tecnología y futuro.

\section{COMPARACIÓN DE LOS TRES MODELOS DE INNOVACIÓN}

En la tabla 1 hemos resumido la descripción de los tres modelos de innovación previamente analizados (la gestión cívica, los comunes urbanos y los ateneos de innovación democrática), lo que nos sirve para comparar sus diferencias y similitudes.

Tabla 1. Resumen casos de estudio

\begin{tabular}{|c|c|c|c|}
\hline & Gestión cívica & Comunes Urbanos & Ateneos de innovación democrática \\
\hline Caso & Centro LGTBI & Can Batlló & Canódromo \\
\hline $\begin{array}{l}\text { Entidad } \\
\text { Impulsora }\end{array}$ & $\begin{array}{l}\text { Consejo Municipal } \\
\text { LGTBI }\end{array}$ & $\begin{array}{l}\text { Movimiento vecinal y cooperativo del } \\
\text { barrio de Sants }\end{array}$ & Ayuntamiento de Barcelona \\
\hline Objetivos & $\begin{array}{l}\text { Convertirse en un } \\
\text { espacio } \\
\text { referencia para el } \\
\text { movimiento } \\
\text { asociativo LGTBI de } \\
\text { la ciudad }\end{array}$ & $\begin{array}{l}\text { Dinamizar la reapropiación colectiva } \\
\text { de los antiguos espacios fabriles de } \\
\text { Can Batlló y practicar su autogestión } \\
\text { comunitaria como bienes comunes } \\
\text { urbanos, con el fin de abrirlos en el } \\
\text { barrio y la ciudad, y ponerlos al } \\
\text { servicio de "la construcción de } \\
\text { alternativas al modelo capitalista, } \\
\text { heteropatriarcal y colonialista de } \\
\text { ciudad". }\end{array}$ & $\begin{array}{l}\text { Promover servicios, proyectos y } \\
\text { espacios para la promoción de la } \\
\text { innovación democrática, el uso de } \\
\text { tecnologías libres, la capacitación } \\
\text { digital y la ciencia ciudadana, a fin de } \\
\text { contribuir a la disminución de la brecha } \\
\text { digital entre la ciudadanía y aumentar } \\
\text { la participación a través de la } \\
\text { tecnología. }\end{array}$ \\
\hline Cesión & $\begin{array}{l}\text { Convenio de gestión } \\
\text { cívica }\end{array}$ & $\begin{array}{l}\text { Convenio de cesión a la Asociación } \\
\text { Espacio Comunitario y Vecinal de } \\
\text { Can Batlló }\end{array}$ & Contratación a Colectic SCCL \\
\hline Gobernanza & $\begin{array}{l}\text { Consejo del Centro y } \\
\text { Comisión de } \\
\text { Seguimiento. }\end{array}$ & $\begin{array}{l}\text { Comisión de Seguimiento y Balance } \\
\text { Comunitario. }\end{array}$ & $\begin{array}{l}\text { Taula Comunitària con entidades del } \\
\text { barrio. } \\
\text { Taula Canòdrom } \\
\text { Decidim el Canòdrom }\end{array}$ \\
\hline Ámbito & $\begin{array}{l}\text { Diversidad sexual y } \\
\text { de género }\end{array}$ & Economía Social y Cooperativa & $\begin{array}{l}\text { democratización de la innovación } \\
\text { digital. }\end{array}$ \\
\hline
\end{tabular}

Fuente: elaboración propia

En primer lugar, se evidencia que los tres casos plantean tres modelos distintos de gobernanza: el centro LGTBI se basa en un modelo de co-gestión centrado en las entidades, mientras Can Batlló se centra en la articulación con movimientos sociales y economía cooperativa con una orientación explícitamente anticapitalista, mientras el Canódromo presenta un modelo mixto que integra las entidades del barrio, los colectivos y proyectos residentes, los colectivos afines y las personas usuarias del espacio a través de la plataforma decidim.canodrom.

En segundo lugar, se evidencia que dos de los modelos utilizan la forma convenio a entidades, que es el instrumento jurídico asociado a la gestión cívica, mientras el Canódromo opera a través de un contrato de adjudicación a una cooperativa. Sin embargo, el desarrollo del modelo de gobernanza seguido por el Canódromo (mesa de entidades, espacio de gestión con los colectivos y abertura a la participación a través de la plataforma decicim.canodrom, permite incluirlo como un espacio de co-gobernanza.

En tercer lugar, se evidencia también que los tres casos elegidos responden a tres áreas políticas que han sido prioritarias para el equipo de gobierno de Barcelona en Comú: la diversidad sexual y 
de género, la promoción de la economía social y colaborativa, y la democratización de la innovación digital, y que ambos operan en el marco conceptual de la colaboración público-comunitaria definido en el Programa de Patrimonio Ciudadano.

Finalmente, cabe destacar por su carácter innovador en el campo de la rendición de cuentas la apuesta de Can Batló por el Balance Comunitario, que permite evaluar "el arraigo territorial, la corresponsabilidad social, la gestión democrática, la participación ciudadana, la orientación a las necesidades humanas, el compromiso con la comunidad y el retorno social" (Castro y Forné, 2021, p. 36).

Tabla 2. Comparación de los Modelos de innovación

\begin{tabular}{|l|l|l|l|l|}
\hline & Actores & Participación & Impacto & Innovación \\
\hline Gestión Cívica & Sector Asociativo & Consorciada & $\begin{array}{l}\text { Empoderamiento del tejido } \\
\text { asociativo-ciudadano en el } \\
\text { territorio }\end{array}$ & Gestión \\
\hline Comunes Urbanos & $\begin{array}{l}\text { Cooperativas y movimientos } \\
\text { sociales }\end{array}$ & Autogestión & $\begin{array}{l}\text { Generación de alternativas } \\
\text { económicas y sociales }\end{array}$ & Social \\
\hline $\begin{array}{l}\text { Democratización de } \\
\text { la innovación digital }\end{array}$ & $\begin{array}{l}\text { Desarrolladores, } \\
\text { ciberactivistas, entidades } \\
\text { ciudadanas }\end{array}$ & Deliberativa & $\begin{array}{l}\text { Capacitación ciudadana en } \\
\text { un uso democrático de la } \\
\text { ciencia y la tecnología. }\end{array}$ & $\begin{array}{l}\text { Digital- } \\
\text { Democrática }\end{array}$ \\
\hline
\end{tabular}

Fuente: elaboración propia

En la tabla 2, hemos identificado los distintos elementos que componen cada modelo de innovación identificando los actores, el modelo de participación, sus respectivos impactos y el modelo de innovación que asociamos. En este sentido, el modelo de gestión cívica se orienta principalmente al sector asociativo (entidades) y adopta un formato de participación consorciado entre entidades y administración que se vehicula a través de comisiones de seguimiento, su principal impacto es el empoderamiento del tejido asociativo del barrio o del sector y la innovación generada se produce de forma prioritaria en las formas de gestión, al constituir una alternativa a la gestión indirecta. El modelo de comunes urbanos tiene como protagonistas principales a los movimientos sociales y al sector de la economía social y solidaria, promoviendo una participación autocentrada que se orienta a la autogestión, en que el mecanismo de rendición de cuentas es el balance comunitario que permite medir el retorno social de la acción desarrollada; en cambio la democratización de la innovación digital y democrática tiene como protagonista los colectivos de creadores y desarrolladores de tecnología (makers, desarrolladores de software libre, ciberactivistas, ciberfeministas....) y promueve un tipo de participación deliberativa y abierta orientada a la democratización de la ciencia y la tecnología, por lo que podemos ubicar su modelo de innovación como digital-democrático.

\section{DISCUSIÓN Y CONCLUSIONES}

En este artículo hemos identificado tres modelos de innovación democrática que se han desarrollado en Barcelona durante el periodo 2015-2019 coincidiendo con el acceso al gobierno de la ciudad Barcelona en Comú: la gestión cívica de equipamientos, la producción de comunes urbanos y el impulso a prácticas de democratización de la innovación digital. Tal y como se ha evidenciado, en su producción han confluido, por una parte, la voluntad política del equipo de gobierno del ayuntamiento de Barcelona por impulsar la gestión cívica y fortalecer diferentes 
prácticas de comunalización de la ciudad y por otra, el impulso del tejido asociativo, de los movimientos sociales y urbanos y del ecosistema de innovación, que ha permitido la coproducción de un nuevo modelo de innovación favorecedor de los procesos de democratización (Tilly, 2007) en tres ámbitos (la gestión pública, la economía urbana y la innovación digital), por lo que podemos incluirlos como prácticas de innovación democrática.

Los tres modelos se distinguen por las diferentes formas de articulación de los actores implicados (entidades, movimientos sociales-economía cooperativa y colectivos de creación digital), por su finalidad (cogestión ciudadana, autogestión comunitaria y democratización de la innovación digital) y por las formas de articulación de la gobernanza (consejo de centro, asamblea y gobernanza digital distribuida a través de la plataforma digital decidim.canodrom). No obstante, los tres modelos participan de un mismo marco de gestión (la colaboración público-comunitaria) que emerge como un modelo de gestión alternativo a la colaboración público-privada que se asocia al paradigma de la nueva gestión pública. En este sentido, podemos adscribir estos tres modelos al contra-movimiento de protección (Polanyi, 1944; 2016) frente a la tendencia a la mercantilización de las instituciones públicas y comunitarias por parte del sector privado.

Los tres casos identificados presentan elementos de interés para el estudio de la innovación democrática. El Centro LGTBI en tanto equipamiento sectorial de ciudad supone una extensión del modelo de gestión cívica que se había desarrollado principalmente en ámbitos territoriales más reducidos. Can Batlló representa una novedosa articulación de diferentes proyectos autogestionados (espacio cultural, cooperativa, escuela, vivienda...) mediante una cesión de uso del espacio incorporando el balance comunitario como mecanismo de rendición de cuentas, y el Canódromo presenta un modelo que busca hibridar el territorio con espacios de creación orientados a democratizar la innovación digital desde la perspectiva de los comunes digitales y la co-creación-difusión de ciencia y tecnología en clave democrática. En los tres casos se observa la existencia de una comunidad que existe previamente y que facilita el desarrollo del proyecto.

En este contexto, el equipo de gobierno del Ajuntament de Barcelona ha actuado como elemento facilitador para la definición de un nuevo modelo de gobernanza a través de la aprobación de un marco normativo que ofrece seguridad jurídica y diseña un nuevo marco conceptual de coproducción de servicios públicos (patrimonio ciudadano, gestión cívica, comunes urbanos), basado en la cooperación público-comunitaria. El interés de este modelo de cooperación reside en que permite establecer una alianza entre la capacidad de autogestión y afirmación de los movimientos sociales con la defensa del interés y el valor público (Moore, 1998) y la co-construcción de nuevos imaginarios que permiten la apertura de nuevos espacios de contienda política (Chatterton, 2010), tal y como se evidencia en el desarrollo de la experiencia del balance comunitario desarrollado en Can Batlló; logrando de esta manera una nueva articulación entre movimiento social e institución pública que no comporta necesariamente una des-radicalización del movimiento ni su integración sistémica, sino la construcción de enclaves que permiten la traducibilidad entre diferentes visiones y posiciones, sin que estas sean reducidas a un común denominador (Stavrides, 2014). En este sentido, podemos considerar que este modelo público-comunitario se alinea con la propuesta de Fraser (2013) de un triple movimiento (mercantilización, protección y emancipación) y la propuesta de establecer una alianza entre emancipación y protección, rompiendo así la relación peligrosa entre neoliberalismo y emancipación que pueden acabar generando involuntariamente ciertas concepciones de la autogestión y los bienes comunes pueden acabar siendo funcionales a los intereses del mercado (Papadimitropoulos, 2018). 
Finalmente, resulta de interés por su carácter innovador el modelo de gobernanza adoptado por el Canòdrom - Ateneu per a la Innovació democrática al tratarse de un modelo híbrido que combina la Taula d'Entitats con representación del tejido asociativo del barrio, la Taula Canòdrom como espacio de participación de los colectivos residentes/afines y la plataforma digital decidim.canodrom que aglutina personas y colectivos usuarios del equipamiento. No obstante, la novedad del equipamiento no permite todavía extraer conclusiones de su funcionamiento, lo que merecería una posterior investigación que evaluará el desarrollo de este formato.

\section{REFERENCIAS BIBLIOGRÁFICAS}

Ajuntament de Barcelona (1998). Text consolidat de la Carta Municipal de Barcelona. https://w9.bcn.cat/A juntament/Documents/CartaMunicipal/CMB\%20text\%20consolidat.pdf

Ajuntament de Barcelona (2015). Model de bases de la gestió cívica d'equipaments, per activitats $i$ serveis municipals de l'Ajuntament de Barcelona (procediment de pública concurrència). Disponible a: https:/ /bop.diba.cat/scripts/ftpisa.aspx?fnew?bop2015\&04/022015010598.pdf\&1

Ajuntament de Barcelona (2016a). Pla Digital de l'Ajuntament de Barcelona. Mesura de govern per a la digitalizació oberta: programari lliure i desenvolupament ágil de serveis de l'administració pública. http s://ajuntament.barcelona.cat/digital/sites/default/files/le_mesuradegovern_v2.pdf

Ajuntament de Barcelona (2016b). Pla estratègic per la Direcció de Recerca, Desenvolupament i Innovació de Participació. https://ajuntament.barcelona.cat/innovaciodemocratica/sites/default/files/documents /planestrategico-direccion_innovacio_v_1_0_cat.pdf

Ajuntament de Barcelona (2017). Programa de patrimoni ciutadà d'ús $i$ gestió comunitàries. https://ajuntament.barcelona.cat/participaciociutadana/sites/default/files/document s/resumprogramapatrimoniciutada_novembre2017.pdf

Ajuntament de Barcelona (2018). Model de Governança de les dades municipals, l'Oficina Municipal de Dades i el delegat de Protecció de Dades. https://w123.bcn.cat/APPS/egaseta/cercaAvancada.do?reqCode=d ownloadFile\&publicacionsId=15791

Bonet-Marti, J. (2012). El territorio como espacio de radicalización democrática. Athenea Digital, 12(1), 15-28. https://doi.org/10.5565/rev/athenead/v12n1.914

Bonet-Marti, J. (2021). Construir gobernanza participativa: redes que (co)producen bienestar. En G. Ubasart, y R. Gomà (coord.), Vidas en transición. Reconstruir la ciudadanía social. (313 - 330). Tecnos.

Barber, B, (1984). Strong Democracy: Participatoy Politics for a New Age. University of California.

Bekkers, V.; Tummers, L. y Voorberg, W. (2013). From public innovation to social innovation in the public sector: A literature review of relevant drivers and barriers. Rotterdam: Erasmus University

Benkler, Y. (2015). La riqueza de las redes. Icaria.

Blanco, I. y Gomà, R. (2016). El municipalisme del bé comú. Barcelona: Icaria

Blanco, I.; Cruz Gallach, H.; Martínez Moreno, R. y Parés, M. (2016). El papel de la innovación social frente a la crisis. Ciudad y Territorio Estudios Territoriales, 48(188), 249-260.

Blanco, I.; Gomà, R. y Subirats, J. (2018). El nuevo municipalismo: derecho a la ciudad y comunes urbanos. Gestión y análisis de políticas públicas, 20,14-28 https://doi.org/10.24965/gapp.v0i20.10491

Brugué, Q. y Gomà, R. (1994). Nous models de gestió i d’organització pública. Revista Catalana de Dret Públic, 18, 207-229 
Cabré, E. y A. André (2018). La Borda: a case study on the implementation of cooperative housing in Catalonia, International Journal of Housing Policy, 18(3), 412-432. https://doi.org/10.1080/1949124 7.2017.1331591

Castells, M. (1997). La era de la información: economía, sociedad y cultura. Alianza Editorial.

Castro, M. y Forné, L. (2021). Patrimonio Ciudadano. Un marco para la colaboración público-comunitaria. La Hidra. https://lahidra.net/wp-content/uploads/2021/05/Informe-Patrimonio-Ciudadano-CAST.pdf

Chatterton, P. (2010). Seeking the urban common: Furthering the debate on spatial justice. City, 14(6), 625-628. https://doi.org/10.1080/13604813.2010.525304

Col.lectiu de mestres tècnics de l'Arcadia (2018). Arcàdia, la força d'existir!Un projecte d'educació en i per a la llibertat responsable. Fòrum: revista d'organització i gestió educativa, 45, 20-24

Davies, J. y Blanco, I. (2017). Austerity urbanism: Patterns of neo-liberalisation and resistance in six cities of Spain and the UK. Environment and Planning, 49(7) 1517-1536 https://doi.org/10.1177/0308518 $\underline{\mathrm{X} 17701729}$

Crouch, C. (2004) Post-democracy. Blackwell Publishers

della Porta, D., (1995). Social Movements, Political Violence and the State. Cambridge University Press.

del Guayo, I., (2004). La gestión de servicios públicos locales. Revista de Administración Pública, 165, 87-133.

Dunleavy, P. y Hood, C. (1995). De la Administración Pública tradicional a la nueva gestión pública. Ensayo sobre la experiencia modernizadora de diversos países desarrollados. Gestión Y Análisis De Políticas Públicas, (3), 105-114. https://doi.org/10.24965/gapp.vi3.42

Federici, S. (2020). Reencantar el mundo. Traficantes de Sueños.

Fraser, Nancy (2013). ¿Triple Movimiento? Entender la política a la luz de Polanyi. New Left Review, 81, 125-139

Fuster, M. (2010). Governance of Online Creation Communities: Provision of Infrastructure for the Building of Digital Commons [Tesis doctoral, European University Institute]. European University Institute https:/ /cadmus.eui.eu/handle/1814/14709

Fuster, M; Subirats, J.; Berlinguer, M.; Martínez, R. y Salcedo, J. (2015). Procomún digital y cultural libre. ¿Hacia un cambio de época? Icaria.

Gomà, R. y Rebollo, O. (2001). Democracia local y ciudadanía activa: reflexiones en torno a los presupuestos participativos. En J. Font (ed.) Ciudadanos y decisiones públicas. Barcelona: Ariel.

Grau-Solés, M.; Íñiguez-Rueda, L. y Subirats, J. (2011) ¿Cómo gobernar la complejidad? Invitación a una gobernanza urbana híbrida y relacional. Athenea Digital. Revista de Pensamiento e Investigación Social, 11(1), 63-84

Hardin, G. (1968). The Tragedy of the Commons. Science, 162(3859), 1243-1248.

Harvey, D. (2017). Ciudades rebeldes: del derecho de la ciudad a la revolución urbana. Akal.

Hernández-Ascanio, J.; Tirado-Valencia, P. y Ariza-Montes, A. (2016). El concepto de innovación social: ámbitos, definiciones y alcances teóricos. CIRIEC-España, Revista de Economía Pública, Social y Cooperativa, 88, 164-199

Hood, C. (1991). A Public Management for All Seasons? Public Administration, 69, 3-19.

Ibarra, P.; Martí, S. y Gomà, R. (2008). Creadores de democracia radical. Movimientos sociales y redes de políticas públicas. Icaria.

Ibarra, P.; Martí, S.; Gomà, R. y González, R. (2018). Movimientos sociales y derecho a la ciudad. Creadoras de democracia radical. Icaria.

Klijn, E.H. (2012). Public Management and Governance: a comparison of two paradigms to deal with modern complex problems. En D. Levi Faur (ed) The handbook of governance. Oxford University Press: 201-214 
Laval, C.y Dardot, P. (2015). Común. Ensayo sobre la revolución en el siglo XXI. Gedisa editorial.

Levesque, B. (2013). Social Innovation in Governance and Public Management Systems: Toward a New Paradigm? En F. Moulaert; D. MacCallum; D. Mehmood y A. Hamdouch (eds.) The International Handbook. On Social Innovation Collective Action, Social Learning and Transdisciplinary Research. Edward Elgar Publishers

Mayntz, R. (1993). Governing Failures and the Problem of Governability: some Comments on a Theoretical Paradigm. En J. Kooiman (ed.). Modern Governance: New Government-Society Interactions (pp-9-20). Sage.

Mair, P. (2013). Gobernando el vacío: La banalización de la democracia occidental. Alianza Editorial.

Méndez, A., Hamou, D., y Aparicio, M. (2021). Códigos comunes urbanos: Herramientas para el devenir común de las ciudades. Icaria

Moore, M. H. (1998). Gestión estratégica y creación de valor en el sector público. Paidós Estado y Sociedad.

Observatori Metropolità de Barcelona (2014). Comuns urbans a Barcelona. Pràctiques de defensa, cura, reapropiació i gestió comunitària. http://bcncomuns.net/wp-content/uploads/2014/06/Conclusions _OMB_CAT.pdf

Ostrom, E. (1990). Governing the commons. The evolution of institutions for collective action. Cambridge University Press. https://doi.org/10.1017/CB09780511807763

Pascual, J. M. (2016). Las ciudades ante el cambio de era. Editorial Hacer.

Papadimitropoulos, V. (2018). Reflections on the Contradictions of the Commons. Review of Radical Political Economics, 50(2), 317-331. https://doi.org/10.1177/0486613417735660

Pastor Albaladejo, G. (2014). Co-creación de servicios públicos. El caso de los servicios de asistencia personal de la Comunidad de Madrid. Documento para su presentación en el V Congreso Internacional en Gobierno, Administración y Políticas Públicas GIGAPP-IUIOG. Instituto Nacional de Administración Pública (Madrid, España) 29, 30 septiembre y 1 octubre 2014. https://eprints.ucm.es/id/eprint/4279 3/1/2014_Pastor-Ponencia\%20GIGAPP-Cocreaci\%C3\%B3n.pdf

Peña-López, I. (2019). Convirtiendo participación en soberanía: el caso de decidim.barcelona. Editorial Huygens.

Plataforma Gestió ciutadana (2012). Presentació de la Plataforma Gestió Ciutadana de Barcelona. https://c asaorlandai.cat/wp-content/uploads/2015/02/presentacic3b3-pgc-1.pdf

Polanyi, K. (1944; 2016). La gran transformación. Virus Editorial.

Pont Vidal, J. (2016). Modelos innovadores de administración y gestión pública: Hacia la emergencia de nuevos paradigmas. Gestión y Análisis de Políticas Públicas, (16), 6-26. https://doi.org/10.24965/gap p.v0i16.10364

Pradel, M. y García, M. (eds.) (2018). El momento de la ciudadanía. Innovación social y gobernanza urbana. Madrid: Libros la Catarata.

Rifkin, J. (2014). La sociedad de coste marginal cero: el internet de las cosas, los bienes comunes y el eclipse del capitalismo. Paidós Ibérica.

Sánchez Belando, M.V. (2015). Las Políticas culturales de proximidad en el paradigma de la ciudad creativa: el caso del programa de centros cívicos en la ciudad de Barcelona, Política y Sociedad, 52(1), 125-152. https://doi.org/10.5209/rev_P0SO.2015.v1.n52.45431

Sánchez Belando, M.V. (2021). Si no ho feu vosaltres, ho farem nosaltres. Can Batlló como caso de innovación social dentro de la gobernanza barcelonesa [Tesis doctoral, Universidad de Barcelona]. Tesis Doctorals en Xarxa https://www.tesisenred.net/handle/10803/672480\#page=1 
Scharpf, F. W. (1994). Games real actors could play: positive and negative coordination in embedded negotiations. Journal of Theoretical Politics, 6, 27-53. https://doi.org/10.1177/09516928940060010 $\underline{02}$

Sousa Santos, B. (2003). Democracia y participación: El caso del presupuesto participativo de Porto Alegre. Ediciones El Viejo Topo.

Stavrides, S. (2014). Emerging common spaces as a challenge to the city of crisis. City, 18(4-5), 546-550. $\underline{\mathrm{h}}$ ttps://doi.org/10.1080/13604813.2014.939476

Subirats, J. (2011). Otra sociedad, ¿otra política?. Icaria.

Subirats, J. (2016) El poder de lo próximo. Madrid: La Catarata

Swyngedouw, E. (2005), Governance innovation and the citizen: The Janus face of governance-beyond-theState, Urban Studies, 42(11): 1991-2006. https://doi.org/10.1080/00420980500279869

Tilly, C. (2007). Democracy. Cambridge University Press.

XES (2021). Guia de preguntes del Balanç Comunitari 2021. Xarxa d'Economia Social. https://mercatsocial.xes.cat/wp-content/uploads/sites/2/2021/05/Guia-de-preguntes-Balan \%C3\%A7-Comunitari-2021.pdf

Weber, M. (1922/2014). Economía y sociedad. Fondo de Cultura Económica.

NOTAS

* Jordi Bonet Martí es profesor lector del Departamento de Sociología de la Universitat de Barcelona. Doctor en Psicología Social y máster en gestión de la comunicación política. Ha investigado sobre participación ciudadana, políticas urbanas, movimientos sociales y estudios de género.

Contacto: jordi.bonet@ub.edu

** Mercedes Serrano Miguel es profesora lectora en la Escola de Treball Social de la Universitat de Barcelona. Es doctora en Antropología Social y ha investigado en participación social en la salud, desigualdad y salud colectiva. Contacto: mercedes.serrano@ub.edu 